ORIGINAL ARTICLE

\title{
Characterization of HIV-1 Integrase Gene and Resistance Associated Mutations Prior to Roll out of Integrase Inhibitors by Kenyan National HIV-Treatment Program in Kenya
}

\author{
Mabeya Sepha ${ }^{1 *}$, Nyamache Anthony ${ }^{2}$, Ngugi Caroline ${ }^{1}$, Nyerere Andrew ${ }^{1}$, \\ Lihana Raphael ${ }^{3}$
}

\footnotetext{
OPEN ACCESS

Citation: Mabeya Sepha, Nyamache Anthony, Ngugi Caroline, Nyerere Andrew, Lihana Raphael. Characterization of HIV-1 Integrase Gene and Resistance Associated Mutations Prior to Roll out of Integrase Inhibitors by Kenyan National HIV-Treatment Program in Kenya. Ethiop J Health Sci. 2020;30(1):37.doi: http:// dx.doi.org/ 10.4314/ejhs.v30i1.6

Received: August 23, 2019

Accepted: October 05, 2019

Published: January 1, 2020

Copyright: (C) 2020 Mabeya S., et al.

This is an open access article distributed under the terms of the Creative Commons Attribution License, which permits unrestricted use, distribution, and reproduction in any medium, provided the original author and source are credited.

Funding: Africa-ai-Japan

Competing Interests: The authors declare that this manuscript was approved by all authors in its form and that no competing interest exists.

Affiliation and Correspondence:

${ }^{1 *}$ Department of Medical Microbiology, school of Biomedical Sciences, Jomo Kenyatta University of Agriculture \&

Technology, Nairobi, Kenya

${ }^{2}$ Department of Biochemistry

Microbiology \& Biotechnology, School of

Pure \& Applied Sciences, Kenyatta

University, Nairobi, Kenya

${ }^{3}$ Centre for Virus Research, Kenya

Medical Research Institute, Nairobi, Kenya

*Email: sephakebira@gmail.com
}

\section{ABSTRACT}

BACKGROUND: Antiretroviral therapy containing an integrase strand transfer inhibitor plus two Nucleoside Reverse Transcriptase inhibitors has now been recommended for treatment of HIV-1-infected patients. This thus determined possible pre-existing integrase resistance-associated mutations in the integrase gene prior to introduction of integrase inhibitors combination therapy in Kenya.

METHODS: Drug experienced HIV patients were enrolled at Kisii Teaching and Referral in Kenya. Blood specimens from (33) patients were collected for direct sequencing of HIV-1 polintegrase genes. Drug resistance mutations were interpreted according to the Stanford algorithm and phylogenetically analysed using insilico tools.

RESULTS: From pooled 188 Kenyan HIV integrase sequences that were analysed for drug resistance, no major mutations conferring resistance to integrase inhibitors were detected. However, polymorphic accessory mutations associated with reduced susceptibility of integrase inhibitors were observed in low frequency; M50I (12.2\%), T97A (3.7\%), S153YG, E92G (1.6\%), G140S/A/C (1.1\%) and E157Q (0.5\%). Phylogenetic analysis (330 sequences revealed that $H I V-1$ subtype $A 1$ accounted for majority of the infections, 26 (78.8\%), followed by $D, 5$ (15.2\%) and C, 2 (6\%).

CONCLUSION: The integrase inhibitors will be effective in Kenya where HIV-1 subtype A1 is still the most predominant. However, occurring polymorphisms may warrant further investigation among drug experienced individuals on dolutegravir combination or integrase inhibitor treatment

KEYWORDS: integrase, dolutegravir, mutations, resistance 


\section{INTRODUCTION}

By 2017, it was estimated that about 20.9 million people living with HIV were on Antiretroviral Therapy (ART) worldwide (1), with estimated 25 million expected to receive ART by 2020 (2). However, according to the Kenyan national HIV estimates, 1,517701 people were living with HIV, of whom 897,644 were on treatment with antiretroviral therapy with 620,057 still in need (3).Since the introduction, free ART in Kenya, HIV-1 associated morbidity and mortality have significantly reduced (4). These drugs suppress viral replication hence reduce viral loads thus helping in maintaining the immune system. However, these benefits could be limited with development of drug resistance leading to treatment failure. Previous studies conducted in Kenya have continuously to show an increasing trend in HIV drug resistance to NNRTI, NRTI and PIs (5-7) hence the need to revise and introduce new classes of drugs in the treatment program. The integrase inhibitors (raltegravir, dolutegravir and elvitegravir) are the latest antiretroviral (ARV) drugs that have been developed and approved for treatment of HIV-1 infections (8). INSTI-based regimens are now the recommended and preferred first-line ART for the treatment of HIV-1 infection in ART-naïve patients due to their favorable side effect profile, limited drug-drug interactions, and its effectiveness in viral suppression (6). The Kenya National AIDS Control Council Programme (NASCOP) revised its treatment guidelines and adopted dolutegravir, an InSTI drug, as first-line drug. For better patient's response to treatment with viral suppression, this approach was used as a way towards implementation of ambitious 90-90-90 UNAIDS strategic to end the AIDS epidemic by 2020. The Ministry of Health has already started to implement these guidelines with Nairobi County as a pilot as they roll out the program to the rest of the country. Kenya is therefore among the first African countries to initiate this program in both drug naïve and experienced patients (9). Previous studies have confirmed that dolutegravir is very effective among drug naïve patients. Due to its low genetic barrier, it is easily resisted with development of drug resistance associated mutations (7). This study was, therefore, appropriate in evaluating any possible preexisting HIV drug resistance against INSTI in order to guide its implementation across the country.

\section{METHODS AND PATIENTS}

A cross-sectional pilot study was conducted and stratified approach used in recruiting HIV infected individuals originating from different geographical parts of the Kisii County but enrolled at HIV comprehensive clinic at Kisii level five teaching referral hospital. Approximately, $5 \mathrm{ml}$ venous blood was drawn from consenting participants and used for the analysis. Demographical data on age, gender and ARVs status was obtained through administered structural questionnaire. The study was approved by the Ethical Committee of Kenyatta University. The study was conducted according to the criteria set by the declaration of Helsinki and each subject signed informed consent before participating in the study.

Viral DNA Extraction and amplification: Viral DNA was extracted from collected blood using QIAamp MinElute Virus kit (Qiagen Inc., Valencia, CA) according to the manufacturers' instructions. A part of the partial HIV-1 pol gene covering $288 \mathrm{bp}$ of the integrase (corresponding to 4493-4780 nt in HIV-1 HXB2) was amplified by nested polymerase chain reaction (PCR) using specific primers. These primers were Unipol5 ( ${ }^{1}$ TGGGTACCAGCACACAAAGGAATAGGA GGAAA-3 $\left.{ }^{1}\right) \quad$ Unipol6 (515 CCACAGCTGATCTCTGGCCTTCTCTGTAATA GACC- $\left.3^{1}\right)$ in the first round and Unipoll (5 ${ }^{1}$ AGTGGATTCATAGAAAGCAGAAGT-3' ${ }^{1}$, Unipol2

$\left(5^{1-}\right.$ CCССТАТTCCTTCСССТTCTTTTAAAA-3 ${ }^{1}$ ) in the second round respectively. Amplification conditions were 1 cycle of $95^{\circ} \mathrm{C}, 10 \mathrm{~min}, 35$ cycles of $95^{\circ} \mathrm{C}, 30 \mathrm{~s}, 45^{\circ} \mathrm{C}, 30 \mathrm{~s}, 72^{\circ} \mathrm{C}, 60 \mathrm{~s}$ and final extension for $72^{\circ} \mathrm{C}$ for $7 \mathrm{~min}$ (6). Amplified products were confirmed by ethidium bromide stained gel electrophoresed and visualised under ultraviolet light and directly sequenced (Applied Biosystems, Foster City, CA) using the manufacturer's protocol as previously described (56 ). The generated sequences were analysed for the presence of resistance associated substitution/mutations using Stanford HIV drug 
resistance database (http://hivdb.stanford.edu/) and mutations compared to those of IAS drug resistance algorithm.

$\mathrm{CD4}^{+} \mathrm{T}$ cell counts: Baseline $\mathrm{CD}^{+}{ }^{+} \mathrm{T}$ cell count was performed using a FACSCalibur flow cytometer (Becton-Dickinson, NJ) equipped with automated acquisition and analysis software according to the manufactures instructions. The ART status of the study subjects were determined prior to recruitment and baseline $\mathrm{CD} 4^{+}$counts determined.

Phylogenetic analysis: The generated sequences were phylogenetically analysed using MEGAx software. Generated sequences were aligned and joined together with reference sequences using Clustal W (version 1.6.6) and Neighbour-joining softwares respectively. Phylogenetic tree was then inferred by Tree View software (version 1.6.6) at bootstrap resampling (1000 data sets) for statistical robustness (12).

Genotypic drug resistance analysis: The generated sequences from this study (33) were pooled together with all other Kenyan integrase sequences from the Gen Bank (155) and analysed for possible drug resistance associated mutations. Genotypic drug resistance in the HIV-1 polintegrase region was defined as the presence of one or more resistance-related mutations, as specified by the consensus mutation figures of the International AIDS Society-USA $(6,14)$.
Ethical approval and consent participate: This study was approved by the Ethical Committee of Kenyatta University and permission by Hospital ethical management committee. The study was conducted according to the criteria set by the declaration of Helsinki and each subject signed an informed consent before participating in the study.

\section{RESULTS}

Study populations: A pilot study was conducted targeting HIV infected patients attending HIV comprehensive clinics of Kisii Teaching and Referral Hospital. A total of 33 consenting drug experienced individuals consisting females (20) and males (13) were recruited into the study during the period between January and July 2018. The average age of the studied subjects was 47.8 years for males and 27 years for females. The ages ranged from 14 to 73 years. From the CD4 counts findings, the majority of the participants had their CD4 counts above 500 Cells $/ \mathrm{mm} 3$ (Table 1). The CD4 lymphocyte count ranged from 220 to 998 Cells $/ \mathrm{mm} 3$ with average of 522 Cells $/ \mathrm{mm} 3$. We compared if gender and age had any relationship with the CD4 counts. From the analysis, neither gender $(p=0.576)$ nor age $(p=0.844)$ had significant difference in relation to levels of CD4 counts.

Table 1. Characteristics of Kenyan HV infected patients in Kisii prior to introduction of integrase inhibitors.

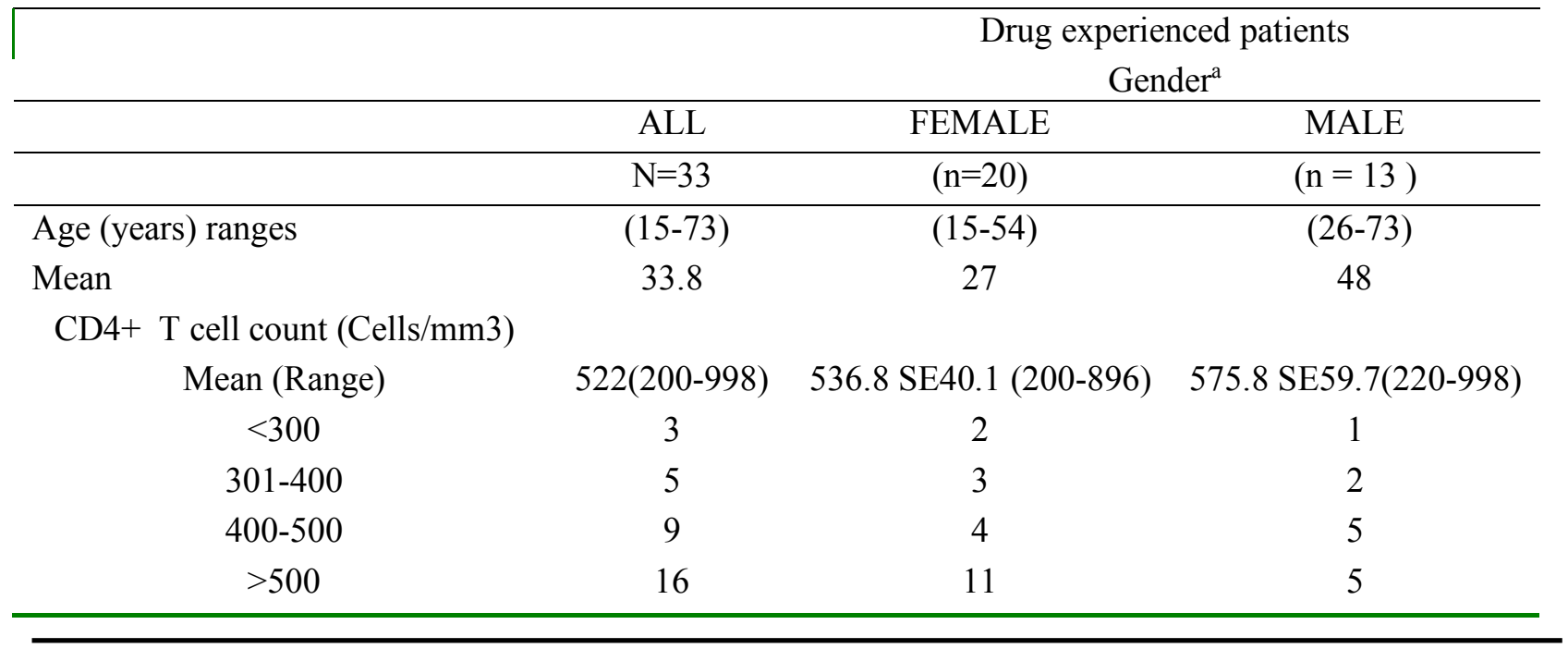

DOI: http://dx.doi.org/10.4314/ejhs.v30i1.6 
Integrase drug resistance: Thirty three (33) sequences from this study were pooled together with 155 Kenyan integrase sequences from the Los Alamos database accessed on May $12^{\text {th }}, 2019$ (Supplementary data) and analysed for drug resistance mutations. Drug resistance was defined according to the Stanford HIVdb's (http://hivdb.stanford.edu/) as guided by International AIDS Society-USA. All 188 sequences were analysed for HV-1 drug resistance. From analysis of these integrase sequences, no primary mutations $(\mathrm{Y} 143 \mathrm{R}=\mathrm{C}=\mathrm{H}, \mathrm{Q} 148 \mathrm{H}=\mathrm{R}=\mathrm{K}$, and $\mathrm{N} 155 \mathrm{H}=\mathrm{S})$ associated with reduced susceptibility to the integrase inhibitors Raltegravir and Elvitegravir were detected. However, about $20 \%$ of the analysed sequences had mutations associated with reduced susceptibility to INSTI. These mutations were M50I (12.2\%), T97A $(3.7 \%), \quad \mathrm{S} 153 \mathrm{YG}, \quad$ E92G (1.6\%), G140S/A/C $(1.1 \%)$ and E157Q (0.5\%). In addition, these sequences had also naturally occurring polymorphisms. HIV subtypes were analysed for any possible relationship with occurrence of HIV-1 drug mutations. From the analysis, there is no relationship between HIV subtype and occurrence of drug resistance (Table 2).

HIV-1: subtype distribution: Phylogenetic analyses of the (33) sequences revealed that the majority of the sequences belonged to A1: 26 $(78.8 \%)$ followed by D: $5(15.2 \%)$ and then C: 2(6\%) (Figure 1).

Table 2: Frequency of HIV-1 integrase drug resistance associated mutations found in Kenyan HIV-1 integrase sequences.

\begin{tabular}{ccc}
\hline Integrase Mutations & Frequency $\mathbf{1 8 8}(\mathbf{n})$ & \% \\
\hline none & 165 & 87.8 \\
M50I & 23 & 12.2 \\
\hline E157Q & 1 & 0.5 \\
L74M/I & 3 & 1.6 \\
T97A & 7 & 3.7 \\
G140S/A/C & 2 & 1.1 \\
S153YG & 1 & 1.6 \\
E92G & 1 & 1.6 \\
E92V & 1 & 1.6 \\
\hline
\end{tabular}


HIV-Integrase Gene and Resistance...

Mabeya S. et al.

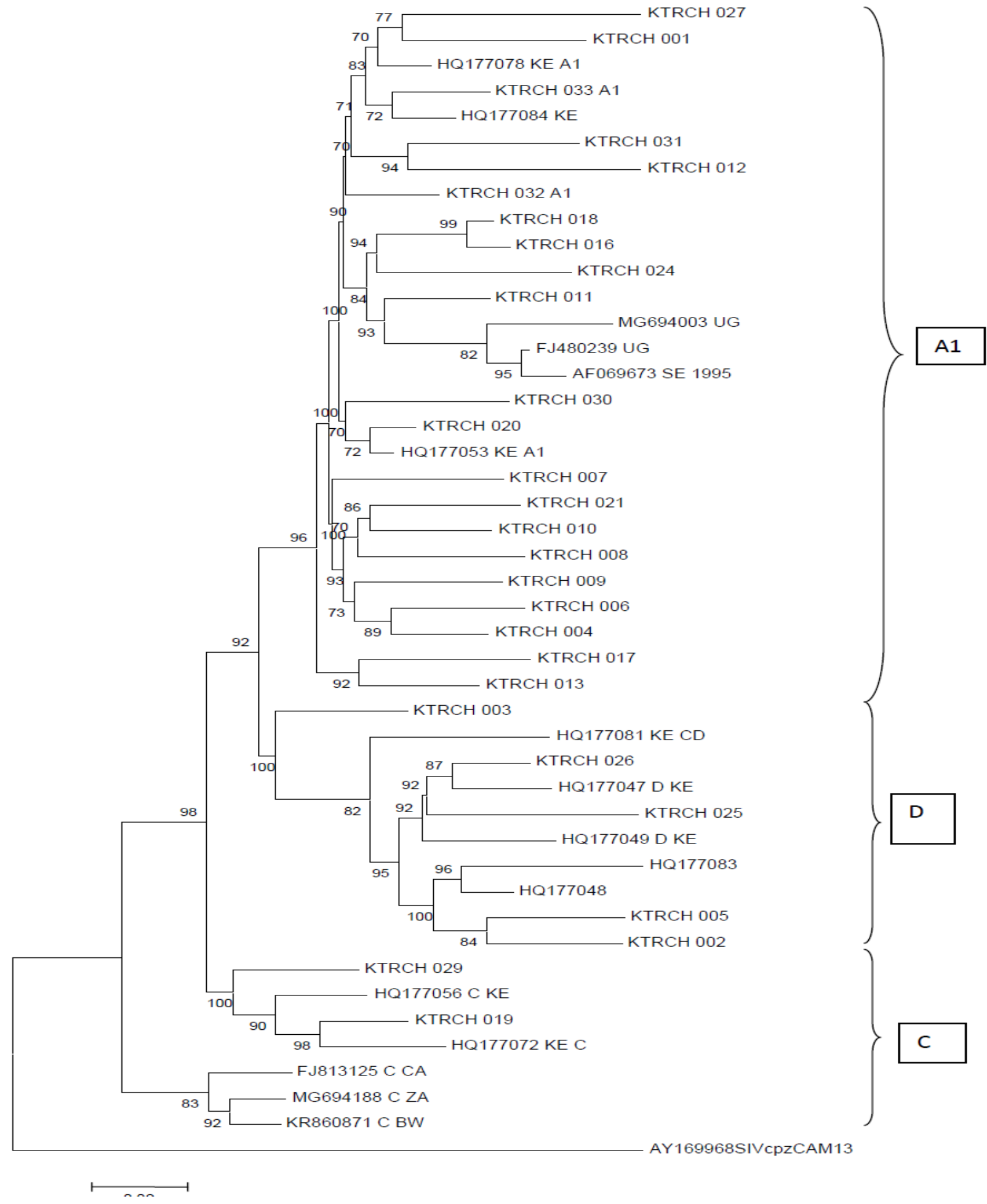

Figure 1: Phylogenetic tree of the HIV -1 pol-integrase sequences from Kisii region in relation to reference sequences from the Los Alamos HIV database

DOI: http://dx.doi.org/10.4314/ejhs.v30i1.6 


\section{DISCUSSION}

In the present study, HIV-1 strains sampled in Kisii were characterised based on pol-integrase region. Like in the rest of parts of the country, the majority of the analysed sequences belonged to HIV-1 subtype A1 with HIV-1 subtype C being the least. These findings agree with previous studies on the predominance of HIV1 subtype A1 in Kenya $(6,10,16)$. Sequences belonging to HIV-1 subtype A1 clustered with sequences from Kenya, Uganda and Sweden. The fact that these sequences clustered with those from the neighborhood countries, there is a likelihood of them having a common evolutionary origin (16). The sequences aligned mainly with those from Kenya, indicating possible Kenyan origin. The sequences that were HIV subtype C clustered with reference sequences from Kenya, Zambia, Canada and Botswana. The HIV-1 subtype D sequences clustered with reference sequences from Kenya (5-6,16). From these findings, the commonly detected subtypes A1, C and D were detected though with no recombinants. This does not rule out possible circulation of recombinants based possible intratransmission of these viral strains within Kenya borders. Nevertheless, based on amplified small gene fragment, this may not have allowed detection of possible viral recombinants. These findings confirm that the HIV transmission occurs within Kenyan borders. There is compelling evidence that variation in HIV-1 subtypes could influence the development of drug resistance and the susceptibility to certain antiretroviral drugs (1718). Certain integrase mutations do differ according to subtype in response to integrase inhibitor efficacy (19). From our analysis, however, there was no significant relationship of HIV-1 subtypes and occurrence of mutations.

Three INSTIs, drugs, RAL, DTG and EVG are new class of drugs that have been approved for clinical use by the FDA and European Medicines Agency (8). These drugs have shown to be highly efficient for the treatment of both ARV-naïve and experienced individuals even with preexisting drug resistance or treatment complication (20). Based on these findings, several countries including Kenya have revised their treatment guideline and adopted INSTIs in their treatment guidelines. Kenya for instance, by the year 2018, started rolling out this guideline with its implementation being piloted in Nairobi before rolling it out to the rest of the country.

In Kenya, provision of free antiretroviral (ARVS) to the public began in 2003. This has significantly promoted its access to AIDS patients (7). With current WHO guideline "diagnose and treat", the demand for ARVs has significantly increased. In this study, we analysed for any possible preexisting drug resistance mutations prior to INSTIs introduction. From our analysis, no major or minor mutations associated with reduced susceptibility to INSTIs dolutegravir, Raltegravir and Elvitegravir or Bictegravir were detected (Table 2). According to previous studies and the fact that INSTIs have not yet been introduced in Kenya, polymorphic accessory mutations that are associated with reduced susceptibility to Raltegravir and Eletegravir were detected. These mutations (Table 2) occurred in low frequencies which suggests that the INSTIs could be effective. Based on high genetic barrier to resistance in dolutegravir, detected mutations were only those associated with raltegravir and eletegravir that have a low genetic barrier. These mutations being polymorphic in drug sensitive HIV-1, these mutations could naturally enhance viral fitness and virulence (20). This study confirms previous findings that detected these naturally occurring polymorphisms, suggesting possible adaptation to immune or drug pressures (6). These mutations could occur even at positions that could be linked to drug resistance to either dolutegravir raltegravir or eletegravir (6). Polymorphic mutations were detected among the analysed sequences (Table 2). These mutations are known to be associated with potential high level of resistance to RAL and EVT, which in combination with major mutation could synergistically reduce susceptibility INSTIs. Even though dolutegravir could be preferred regimen, its efficacy could be limited with development drug resistance as INSTIs are being rolled out for the management of HIV/AIDS. Based on this development, continued surveillance of INSTI resistance in the country is invertible.

Despite being a pilot study, no major mutations associated with INSTIs drug resistance were detected. However, naturally occurring 
polymorphisms were detected at low frequency. Such mutations have not been associated with any drug resistance. These findings, therefore, confirm that treated HIV-1 infected Kenyans are likely to benefit from INSTI-based drug regimens, especially at this time when there is increasing trends of HIV drug resistance against reverse transcriptase inhibitors, which are currently being used in the country. Like the rest parts of the Country, HIV-1 subtype A1 remains the most predominant circulating subtype.

\section{REFERENCES}

1. UNAIDS. Fact Sheet-Latest Statistics on the Status of the AIDS Epidemic. 2017. (Available from: http://www.unaids.org/en/resources/factsheet) (Accessed 07 December 2018).

2. Estill, J., Ford N., Salazar-Vizcaya, L., Haas, A. D., Blaser, N., Habiyambere, V et al. The need for second-line antiretroviral therapy in adults in sub-Saharan Africa up to 2030: a mathematical modelling study. The Lancet $H I V, 2016$;3(3): e132-e139.

3. National AIDS and STI Control Program (NASCOP) Kenya Ministry of Health. (2018). Guidelines on Use of Antiretroviral Drugs for Treating and Preventing HIV in Kenya.

4. Mukui, I. N., Williamson, J., Wamicwe, J. N., Vakil, S., Katana, A., \& Kim, A. A. Rates and predictors of non-adherence to antiretroviral therapy among HIV-positive individuals in Kenya: results from the second Kenya AIDS indicator survey, 2012. PloS one, 2016;11(12): $\mathrm{e} 0167465$.

5. Lihana, R.W., Khamadi, S.A., Lubano, K., Lwembe, R., Kiptoo, M.K., Lagat, N., et al. HIV type 1 subtype diversity and drug resistance among HIV type 1-infected Kenyan patients initiating antiretroviral therapy. AIDS research and human retroviruses, 2009;25(12):1211-1217.

6. Nyamache, A. K., Muigai, A. W., Nganga, Z., \& Khamadi, S. A. HIV Type 1 genetic diversity and naturally occurring polymorphisms in HIV type 1 Kenyan isolates: implications for integrase inhibitors. AIDS research and human retroviruses, 2012;28(8):933-936.

7. Koigi, P., Ngayo, M. O., Khamadi, S., Ngugi, C., \& Nyamache, A. K. HIV type 1 drug resistance patterns among patients failing first and second line antiretroviral therapy in Nairobi, Kenya. BMC research notes, 2014;7(1):890.

8. Alaoui, N., El Alaoui, M. A., Touil, N., El Annaz, H., Melloul, M., Tagajdid, R., et al. Prevalence of resistance to integrase strandtransfer inhibitors (INSTIs) among untreated HIV-1 infected patients in Morocco. BMC research notes, 2018;11(1): 369.

9. Mesplede, T., \& Wainberg, M. A. Integrase strand transfer inhibitors in HIV therapy. Infectious diseases and therapy, 2013;2(2):83-93.

10. Lihana, R.W., Khamadi, S.A., Lubano, K., Lwembe, R., Kiptoo, M.K., Lagat, N., et al. HIV type 1 subtype diversity and drug resistance among HIV type 1-infected Kenyan patients initiating antiretroviral therapy. AIDS research and human retroviruses, 2009;25(12): 1211-1217.

11. Brado, D., Obasa, A. E., Ikomey, G. M., Cloete, R., Singh, K., Engelbrecht, S., et al. Analyses of HIV-1 integrase sequences prior to South African national HIV-treatment program and available of integrase inhibitors in Cape Town, South Africa. Scientific reports, 2018;8(1):4709

12. Kimura M. A simple method for estimating evolutionary rates of base substitution through comparative studies of nucleotide sequences. J Mol Evol, 1980; 16:111-120.

13. Saitou $N$ and Nei M.The neighbour-joining method: a new method for reconstructing phylo-genetic trees. Molecular Biology and Evolution, 1987;4: 406-425.

14. Johnson, V. A., Brun-Vézinet, F., Clotet, B., Gunthard, H. F., Kuritzkes, D. R., Pillay, D \& Richman, D. D. Update of the drug resistance mutations in HIV-1: 2009. Top HIV Med, 2009;17(5):138-145

15. Msimanga, P. W., Vardas, E., \& Engelbrecht, S. HIV-1 diversity in an antiretroviral 
treatment naïve cohort from Bushbuckridge, Mpumalanga Province, South Africa. Virology journal, 2015;12(1).

16. Khamadi, S.A., Ochieng, W., Lihana, R.W., Kinyua, J., Muriuki, J., Mwangi, J., et al. HIV type 1 subtypes in circulation in northern Kenya. AIDS Research \& Human Retroviruses, 2005;21(9):810-814.

17. Lessells, R. J., Katzenstein, D. K., \& De Oliveira, T. Are subtype differences important in HIV drug resistance? Current opinion in virology, 2012;2(5):636-643.

18. Bar-Magen T, Donahue DA, McDonough EI, Kuhl BD, Faltenbacher VH, Xu H, Michaud $\mathrm{V}$, et al. HIV-1 subtype $\mathrm{B}$ and $\mathrm{C}$ integrase enzymes exhibit differential patterns of resistance to integrase inhibitors in biochemical assays. AIDS. 2010;24(14): 2171-2179.
19. Xiao, H., Xue, Y., Gu, S., Wang, J., Sun, H., $\& \mathrm{Lu}, \mathrm{H}$. Efficacy and safety of antiretroviral regimens including raltegravir to treat HIVinfected patients with hemophilia. Bioscience trends, 2016;10(1):42-46.

20. Meixenberger K, Yousef KP, Smith MR, Somogyi S, Fiedler S, Bartmeyer B, Hamouda $\mathrm{O}$, et al. Molecular evolution of HIV-1 integrase during the 20 years prior to the first approval of integrase inhibitors. Virol $J$; 2017;14:(1)23.

21. Arruda, L. B., Fonseca, L. A. M., Duarte, A. J., \& Casseb, J. Genetic diversity on the integrase region of the pol gene among HIV type 1-infected patients naive for integrase inhibitors in Sao Paulo City, Brazil. AIDS research and human retroviruses, 2010;26(1):105-107. 